\title{
Relationship between electrical gap after Maze procedure and atrial arrhythmia and ablation outcome after cardiac surgery and concomitant Maze procedure.
}

\author{
Keisuke Suzuki ${ }^{1}$, Koji Miyamoto ${ }^{1}$, Ueda Nobuhiko ${ }^{1}$, Kenzaburo Nakajima ${ }^{1}$, Tsukasa \\ Kamakura $^{1}$, Mitsuru Wada ${ }^{1}$, Kennichiro Yamagata ${ }^{1}$, Kohei Ishibashi ${ }^{1}$, Yuko Inoue ${ }^{1}$, \\ Takashi Noda ${ }^{1}$, Satoshi Nagase ${ }^{1}$, Takeshi Aiba ${ }^{1}$, Tomoyuki Yambe ${ }^{2}$, Takashi Kakuta ${ }^{1}$, \\ Naoki Tadokoro ${ }^{1}$, Satsuki Fukushima ${ }^{1}$, Tomoyuki Fujita ${ }^{1}$, and Kengo Kusano ${ }^{1}$ \\ ${ }^{1}$ National Cerebral and Cardiovascular Center \\ ${ }^{2}$ Institute of Development, Aging and Cancer, Tohoku University
}

April 29, 2020

\begin{abstract}
Introduction: There are few reports of the characteristics, mechanisms, and outcomes of atrial tachycardia (AT) and atrial fibrillation (AF) ablation after cardiac surgery and concomitant Maze procedures. This study investigated the mechanisms and long-term outcomes of AT and AF ablation after various Maze procedures, especially we examined in detail whether the arrhythmia after Maze procedure was due to gap or not. Methods and Results: We analyzed 37 consecutive cases with AT and AF after cardiac surgery and Maze procedure between 2007 and 2019. Fifty-nine atrial arrythmias were induced in 37 consecutive cases, and 49 of those atrial arrythmias were mappable ATs. Forty-two ATs was related to the Maze procedure in the 49 mappable ATs (87.5\%). All 37 consecutive cases had residual electrical conductions (gaps) in the Maze lines (88 gaps; $2.4 \pm 1.2$ gaps/patient). Forty-two of 88 gaps (47.7\%) were associated with gap-related ATs. The most common gap-related ATs in this study were peri-mitral atrial flutter in 22 cases. The median follow-up period after ablation was 3.6 \pm 3.2 years (median, 2.1 years; interquartile range, 0.89-6.84). The Kaplan-Meier analysis of freedom from recurrent atrial arrhythmia after Maze procedure was $79.9 \%$ at 1-year follow up and $69.3 \%$ at 4 -year follow up. Conclusions: Reentry was the main mechanism of AT after cardiac surgery and concomitant various Maze procedures, and AT were largely related to the gap in the Maze line between mitral valve anulus and pulmonary vein isolation line. Catheter ablation of AT after various Maze procedures seemed to be effective and safe during long-term follow-up.
\end{abstract}

\section{Introduction}

Atrial tachycardia (AT) and atrial fibrillation (AF) commonly occur after cardiac surgery (CS), and Maze procedure has been used to treat and prevent such arrhythmias. ${ }^{1}$ The Maze techniques have been modified over time. Originally, the cut-and-sew technique has been used. In recent years, energy sources, such as radiofrequency (RF), cryo, and Ultrasound (US), have been introduced into the procedure. ${ }^{2-4}$ Typically, Cox III and Kosakai's Maze procedures use the cut-and-sew technique ${ }^{4}$, Maze IV uses RF energy ${ }^{3}$, and CryoMaze $^{2}$ uses cryo energy, but Maze technique depends on operators and the facilities. Surgical Maze has been recommended for maintaining sinus rhythm, ${ }^{5}$ but the failure of the procedure may lead to persistent atrial arrhythmias, which could result in embolism, heart failure, and fatal events. ${ }^{6-8}$

Previously, the electrophysiological mechanisms of ATs after Maze surgery have been reported by some institutions. ${ }^{3,9}$ However, few detailed studies have been made on whether there is a relationship between gaps and AT/AF after various Maze procedures. The purpose of this study was to investigate the characteristics, 
electrophysiological findings, and outcomes of catheter ablation (CA) of atrial arrhythmias after cut-and-sew, $\mathrm{RF}$, or cryo Maze procedures.

\section{Methods}

\section{Patients}

We retrospectively studied 37 consecutive cases after CS and Maze procedure. These patients underwent CA at the National Cerebral and Cardiovascular Center between 2007 and 2019. CS and Maze procedure were performed in various institutions. All patients had symptomatic AT or AF. This study was approved by the institutional review committee of the National Cerebral and Cardiovascular Center and all patients gave their written informed consent before intervention.

\section{Clinical data collection}

All patient data were retrospectively obtained from our CA database. Age, sex, preoperative and postoperative anti-arrhythmia drug (AAD) use, $\mathrm{CHADS}_{2}$ score, $\mathrm{CHA}_{2} \mathrm{DS}_{2}$-VASc score, body mass index, left ventricular ejection fraction, left atrium (LA) size, serum creatinine, hematocrit, and brain natriuretic peptide of each patient were examined. We reviewed all surgical reports and electrophysiological findings.

\section{Collection of surgical records}

We confirmed the record of CS and the Maze procedure before CA. Cryo ablation using the Cardioblate CryoFlex ${ }^{2}$ (Medtronic Inc., Minneapolis, MN, United States) was performed at -60 to -90 degrees for 60 to 180 seconds, and RF ablation was performed as reported previously using the Cardioblate $^{10}$ (Medtronic) or the Atricure ${ }^{11}$ (AtriCure Inc., Cincinnati, OH, United States).

\section{Electrophysiology and ablation strategy}

Before CA, AAD other than amiodarone were discontinued for at least 5 half-lives. The patients were given anticoagulant medication for over 3 weeks before the procedure. Before LA was ablated, transesophageal echocardiography was performed to confirm that there was no thrombus in the LA. Activated clotting time and sedation protocol were as described previously. ${ }^{12}$ Intracardiac echo was used to perform septal puncture. If AT persisted, we observed voltage characterization and activation wavefronts of the atrium. If a patient was in sinus rhythm, AT or AF was induced by atrial burst pacing with a stimulation cycle length of up to $180 \mathrm{~ms}$ or the local refractory period. Intravenous isoproterenol administration was done as necessary. If AT was not sustained, we looked for the existence of gaps after the Maze procedure. When performing CA, we use an ablation catheter with RF energy (NAVISTAR; Biosense Webster, Diamond Bar, California, USA, THERMOCOOL SMARTTOUCH SF; Biosense Webster or TactiCath SE; Abbott, St Paul, MN, USA) at 15-50 W for 5-60 seconds. All ablation lines were confirmed to be bi-directional blocks. Finally, the aforementioned stimulation protocol was performed to confirm the non-inducibility of AT or AF as an endpoint.

\section{Mapping systems}

A three-dimensional (3D) mapping system as the CARTO system (Biosense Webster) or the EnSite system (Abott) was used. A 7-Fr 20-electrode catheter (BeeAT, Japan Lifeline Co. Ltd, Tokyo, Japan) was placed around the tricuspid valve annulus with the distal poles in the coronary sinus. Following access to the LA, 3D geometry was created with a circular mapping catheter (LASSO; Biosense Webster or Inquiry Optima; Abott) or high-resolution catheter (PENTARAY; Biosense Webster or Advisor HD Grid; Abott). Both color isochronal and propagation maps were generated. In the bipolar potential peak-to-peak, the scar area was set as less than $0.1 \mathrm{mV}$, and the low voltage area was set as 0.1 to $0.5 \mathrm{mV} .{ }^{13}$ The gap in this study was defined as the reconnection site where the Maze procedure was performed whether there were slow conduction or not. Space surrounding the pulmonary veins was counted as a gap.

We suspected approximate macroreentrant AT circuits using color-coded 3D electroanatomic activation mapping. ${ }^{12,}{ }^{14}$ Macroreentrant AT was diagnosed when at least 2 atrial pacing sites were within the circuit. 
Sites were considered within the circuit as previously described. ${ }^{\mathbf{9}}$ Non-macroreentrant-AT was defined as occurring from a very small area $(<1 \mathrm{~cm})$ and propagating to the atrium in a centrifugal pattern. ${ }^{15,16}$

\section{Follow-up}

All patients were evaluated at the clinic every 1-3 months using a 12-lead ECG, 24-h Holter monitor, and portable electrocardiograph when patients complained of palpitations; thereafter, they were followed-up annually. Patients whose symptoms recurred were evaluated immediately. Recurrence of tachycardia and complications after $\mathrm{CA}$ were confirmed by outpatient medical records, event monitors, and follow-up via telephone. Recurrence was defined as sustained AT or AF lasting over $1 \mathrm{~min}$.

\section{Data analysis}

Continuous variables were tested for normal distribution using histogram analysis. Data with a normal distribution are presented as the mean \pm standard deviation. Data without a normal distribution are presented as the median and interquartile range (IQR). Categorical variables are expressed as the number and ratio. The chi-square test was used to compare categorical variables. Two-tailed $\mathrm{P}<0.05$ was considered statistically significant. All statistical analyses were performed using IBM SPSS statistics version 25 (IBM Inc., Armonk, NY, USA).

\section{Results}

The median period from consciousness of palpitations to CA was $1.9 \pm 2.6$ years (median, 0.42 years; IQR, 0.17-3.42). Table 1 shows the patients' baseline characteristics. The average age was $69.3 \pm 7.2$ years and 20 (54.1\%) were male. The average LA diameter was $46.9 \pm 7.7 \mathrm{~mm}$.

The types of surgery and outcome are listed in Table $\mathbf{2}$. Among the 37 consecutive cases, the following procedures were performed: CryoMaze, 21; Maze III, 4; Kosakai's Maze, 2; Maze IV, 4; right atrial (RA) Maze, 3; LA Maze, 3. Forty-nine mappable ATs were induced in all cases, of which 42 (85.7\%) were related to the Maze procedure and $47(95.9 \%)$ reached endpoint at first session.

Figure 1 shows the characteristics of 59 atrial arrhythmias, in which 49 were mappable ATs and the remaining 10 were AF. Mappable AT divided into 37 macroreentrant-ATs and 12 non-macroreentrantATs. Macroreentrant-ATs divided into 22 peri-mitral atrial flutter (M-AFL) (20 gap-rerated), 11 cavotricuspid isthmus dependent atrial flutter (T-AFL) (10 gap-rerated), and 4 RA AT (4 gap-rerated). Nonmacroreentrant-ATs were divided into 4 septal AT (2 gap-rerated), 4 LA appendage (LAA) AT (4 gaprerated), 2 LA AT (2 gap- related), and 2 RA AT (0 gap-rerated).

Figure 2 shows all gaps after the Maze procedure. In total, there were 88 gaps (2.4 1.2 gaps/patient), of which $42(47.7 \%)$ were associated with AT. Among the 42 gap-related ATs, 20 (47.6\%) were M-AFL and 11 (26.2\%) were T-AFL. Nineteen of 37 consecutive cases (51.4\%) had gaps in the PV isolation (PVI) line, but only $2(10.5 \%)$ were associated with PV gap reentry AT.

Figure 3 shows representative case 7 . This figure shows lines of the $\mathrm{CryoMaze}^{2}$ procedure performed in our institution, and 3 forms of gap related ATs after CryoMaze procedure.

Table 3 shows the number of AT and AF cases after the Maze procedures. The number of ATs induced was $1.6 \pm 0.8$ per case. Most of ATs were macro reentry tachycardia of the LA. In some cases, LA AT or AF occurred after RA Maze.

Table 4 shows the relationship among the PV gap, the occurrence of PV gap related AT and AF, and the Maze procedure. Almost half of the cases had PV gap and gap-related AT or AF in each Maze procedure. The relationship between cut-and-sew method and the PV gap was not significant different $(\mathrm{P}=0.44)$, and also the relationship between incidence of AF or gap related-AT and cut-and-sew methods $(\mathrm{P}=0.15)$. The incidence of gap related-AT and AF was significantly higher with more gaps in $\mathrm{PV}(\mathrm{P}=0.003)$.

Follow-up 
The median follow-up period after CA was 3.6 \pm 3.2 years (median, 2.1 years; IQR, 0.89-6.84). The KaplanMeier analysis of freedom from atrial arrhythmia after the Maze procedure was $79.9 \%$ at 1-year follow-up and $69.3 \%$ at 4 -year follow-up. AADs were taken after CA in 20 cases (54.1\%). There were no complications throughout the entire period in this study. There were 2 unsuccessful cases in the first session, in which 1 was septal AT and 1 was M-AFL. Twelve cases had recurrence after CA, and 4 of them underwent CA twice. Patients 28, 29, 36, and 38 experienced recurrence (10.8\% of 37 cases), and CA was performed again. All four cases reached the endpoint in the second session, and no recurrence of atrial arrhythmia was observed in Patients 36 and 38 (Fig 4 ). The remaining 8 cases were not willing to undergo CA twice and had a good course of AADs.

\section{Discussion}

This study focused on how gaps formed by Maze procedure affect AF and AT, and to our best knowledge, this focus has never been before. Since the places where gaps are likely to occur have been clarified, it is possible to understand the places where care must be taken to prevent gaps during Maze procedure, and this caution may reduce the recurrence rate of AT and AF. In addition, understanding the difficult-to-treat gaps before catheter ablation may be making it easier to plan treatment.

The main findings of this study were that the distribution of gaps was mainly on the LA and macroreentrantATs also occurred mainly on the LA. Previous studies reported that about two-thirds of macroreentrant-ATs after the Maze procedure occurred on the LA. ${ }^{17-19}$ Our results are similar to previous reports. Of the leftside macroreentrant-ATs, most of the cases were M-AFL and were almost gap-related. In Huo's report, ${ }^{17}$ the incidence of M-AFL in the left-side ATs was about $60 \%$, while in our study, a slightly higher incidence of $78.6 \%$ was found. It has been said that M-AFL catheter ablation was challenging because of convective cooling as a result of coronary sinus blood flow and the thickness of the LA near the mitral valve annulus (MVA). ${ }^{20}$ In our cases, 1 of $22 \mathrm{M}$-AFL cases (4.5\%) failed to be treated at first session, and combination of chemical and RF ablation worked effectively at second session. Although, this study indicated that the gap-related ATs were predominant, but there were some non-gap-related AT. It is conceivable that the arrhythmogenic substrate may have been formed due to the possibility of surgical injury or the load such as ischemia or valvular disease.

Gopinathannair et al. reported that the second common left-side ATs was the roof dependent AFL, ${ }^{13}$ but in our study, there was no case of roof dependent AFL. The reason might be due to that either the roof line or the bottom line in any type of Maze procedure was completed in our study. Additionally, they reported $18 \%$ of PV gap-related ATs were induced. However, in our study, there were only 2 PV gap-related ATs among all 49 mappable ATs (4.1\%). The 2 PV gap-related reentrant ATs were observed in Kosakai's Maze and Maze III cases, indicating cut-and-sew method also could make untransmural lesions that may cause PV gap-related AT.

As previously reported, the number of ATs induced per case was $1.09+-0.35,{ }^{13}$ whereas, in our study, it was $1.6+-0.8$ per case. This incidence may be slightly high. Three or more ATs were induced in patient numbers 7, 8, 21, 25, 27, 28. Patient 7, 21, and 25 underwent the Maze procedure with cryo energy, patient 8 with RF energy, and patient 27 and 28 underwent the Maze procedure with cut-and-sew method. AT and AF may occur due to untransmural lesions that are created in CA or the Maze procedure. ${ }^{21,}{ }^{22}$ Although, Winkle et al reported that compared to the non-cut-and-sew method, the cut-and-sew Maze procedures can form a transmural lesion and result in fewer gaps, ${ }^{15}$ but there are no significant difference of the relationship between gaps and Maze procedure in this study. However, the relationship between AF/PV gap related AT incidence and PV gap were significantly related in this study. In other words, PV gap associated with AT and $\mathrm{AF}$ as previous report. ${ }^{23,}{ }^{24}$ The cut-and-sew technique takes time and effort which may be a burden to the heart, ${ }^{25,} 26$ in contrast to the Maze with cryo or RF energy. To prevent the recurrence of AF/PV gap related AT, it may be necessary to create transmural lesions surround PV using simpler and faster technique.

CryoMaze in our study were all performed in our institute. As Kakuta reported previously, the KaplanMeier analysis showed that the rates of freedom from recurrent AF were $91.9 \%$ at 1 year and $86.1 \%$ at 5 
years. ${ }^{2}$ Over the course of 4 years, the proportion of arrhythmia-free survival after CA following all Maze procedures about $70 \%$ in our study, which was considered similar to that of ordinary AT and AF ablation after Maze procedure. ${ }^{17,} 27$ These data may indicate the combination of Maze procedure and CA may be an effective for regaining sinus rhythm. No intraoperative and postoperative complications were observed in this study. Although the number of cases may be small, it is possible that severe complications such as cardiac tamponade and perforation may not occur because of pericardial adhesions after CS. These facts indicate that AT and AF after any Maze procedure used in this study can be safely and effectively treated.

\section{Limitations}

Our study has several limitations. First, this was a retrospective observational study with small sample size. Although the incidence of AT after the Maze procedure was reported to be $5-15 \%,{ }^{13}$ the frequency of AT after the Maze procedure is unknown because this study included cases from other facilities. In addition, the types of CS and the Maze procedure ${ }^{25}$ such as Maze line and energy sources, varied slightly depending on institutions. Almost half of the cases in this study were treated with CryoMaze ${ }^{2}$ in our institution, but to determine the exact incidence of AT after unified Maze procedure and characteristics of such AT, prospective multicenter studies are needed.

\section{Conclusion}

Reentry was the main mechanism of AT after CS and Maze procedure and was largely related to gap in the Maze procedure line between PVI line and MVA. CA of AT and AF after various Maze procedure seemed to be effective and safe during long-term follow-up.

Acknowledgments: We would like to thank Editage (www.editage.com) for English language editing.

\section{References}

1. Cox JL, Boineau JP, Schuessler RB, et al. Successful surgical treatment of atrial fibrillation. Review and clinical update. JAMA 1991;266:1976-1980.

2. Kakuta T, Fukushima S, Minami K, et al. Contemporary outcomes of the concomitant CryoMaze procedure. Interact Cardiovasc Thorac Surg 2019;29:28-34. DOI:10.1093/icvts/ivz029

3. Gaynor SL, Diodato MD, Prasad SM, et al. A prospective, single-center clinical trial of a modified Cox maze procedure with bipolar radiofrequency ablation. J Thorac Cardiovasc Surg 2004;128:535-542. DOI:10.1016/j.jtcvs.2004.02.044

4. Kosakai Y. Treatment of atrial fibrillation using the Maze procedure: the Japanese experience. Semin Thorac Cardiovasc Surg 2000;12:44-52. DOI:10.1016/s1043-0679(00)70016-5

5. Badhwar V, Rankin JS, Damiano RJ, Jr., et al. The Society of Thoracic Surgeons 2017 Clinical Practice Guidelines for the Surgical Treatment of Atrial Fibrillation. Ann Thorac Surg 2017;103:329341. DOI:10.1016/j.athoracsur.2016.10.076

6. Mueller KAL, Heinzmann D, Klingel K, et al. Histopathological and Immunological Characteristics of Tachycardia-Induced Cardiomyopathy. J Am Coll Cardiol 2017;69:2160-2172. DOI:10.1016/j.jacc.2017.02.049

7. Ellis ER, Josephson ME. Heart failure and tachycardia-induced cardiomyopathy. Curr Heart Fail Rep 2013;10:296-306. DOI:10.1007/s11897-013-0150-z

8. Lloyd-Jones DM, Wang TJ, Leip EP, et al. Lifetime risk for development of atrial fibrillation: the Framingham Heart Study. Circulation 2004;110:1042-1046. DOI:10.1161/01.CIR.0000140263.20897.42

9. Saoudi N, Cosio F, Waldo A, et al. A classification of atrial flutter and regular atrial tachycardia according to electrophysiological mechanisms and anatomical bases; a Statement from a Joint Expert Group from The Working Group of Arrhythmias of the European Society of Cardiology and the North American Society of Pacing and Electrophysiology. Eur Heart J 2001;22:1162-1182. DOI:10.1053/euhj.2001.2658

10. Wisser W, Khazen C, Deviatko E, et al. Microwave and radiofrequency ablation yield similar success rates for treatment of chronic atrial fibrillation. Eur J Cardiothorac Surg 2004;25:1011-1017. DOI: $10.1016 / j . e j c t s .2004 .01 .050$ 
11. Gillinov AM, McCarthy PM. Atricure bipolar radiofrequency clamp for intraoperative ablation of atrial fibrillation. Ann Thorac Surg 2002;74:2165-2168. DOI:10.1016/s0003-4975(02)04484-3

12. Nakasuka K, Miyamoto K, Noda T, et al. "Window Sliding" analysis combined with high-density and rapid electroanatomical mapping: its efficacy and the outcome of catheter ablation of atrial tachycardia. Heart Vessels 2017;32:984-996. DOI:10.1007/s00380-017-0959-6

13. Lo LW, Tai CT, Lin YJ, et al. Progressive remodeling of the atrial substrate-a novel finding from consecutive voltage mapping in patients with recurrence of atrial fibrillation after catheter ablation. $\mathrm{J}$ Cardiovasc Electrophysiol 2007;18:258-265. DOI:10.1111/j.1540-8167.2007.00719.x

14. Gopinathannair R, Mar PL, Afzal MR, et al. Atrial tachycardias after surgical atrial fibrillation ablation: clinical characteristics, electrophysiological mechanisms, and ablation outcomes from a large, multicenter study. JACC Clin Electrophysiol 2017;3:865-874. DOI:10.1016/j.jacep.2017.02.018

15. Winkle RA, Fleming W, Mead RH, et al. Catheter ablation for failed surgical maze: comparison of cut and sew vs. non-cut and sew maze. J Interv Card Electrophysiol 2019;55:183-189. DOI: $10.1007 / s 10840-019-00509-y$

16. Markowitz SM, Thomas G, Liu CF, Cheung JW, Ip JE, Lerman BB. Atrial tachycardias and atypical atrial flutters: mechanisms and approaches to ablation. Arrhythm Electrophysiol Rev 2019;8:131-137. DOI: 10.15420/aer.2019.17.2

17. Huo Y, Schoenbauer R, Richter S, et al. Atrial arrhythmias following surgical AF ablation: electrophysiological findings, ablation strategies, and clinical outcome. J Cardiovasc Electrophysiol 2014;25:725738. DOI: $10.1111 / j c e .12406$

18. Magnano AR, Argenziano M, Dizon JM, et al. Mechanisms of atrial tachyarrhythmias following surgical atrial fibrillation ablation. J Cardiovasc Electrophysiol 2006;17:366-373. DOI:10.1111/j.15408167.2006.00419.x

19. Akar JG, Al-Chekakie MO, Hai A, et al. Surface electrocardiographic patterns and electrophysiologic characteristics of atrial flutter following modified radiofrequency MAZE procedures. J Cardiovascu Electrophysiol 2007;18:349-355. DOI:10.1111/j.1540-8167.2007.00761.x

20. Naniwadekar A, Koruth J. Impact of technique and technology on mitral isthmus ablation. Curr Treat Options Cardiovasc Med 2019;21:46. DOI:10.1007/s11936-019-0752-1

21. Zhan XZ, Liu FZ, Guo HM, et al. Characteristics, mechanism and long-term ablation outcome of atrial tachycardias after mitral valvular surgery and concomitant Cox-MAZE IV procedure. Int Heart J 2019;60:71-77. DOI:10.1536/ihj.18-117

22. Miller MA, d'Avila A, Dukkipati SR, et al. Acute electrical isolation is a necessary but insufficient endpoint for achieving durable PV isolation: the importance of closing the visual gap. Europace 2012;14:653-660. DOI:10.1093/europace/eus048

23. Haissaguerre M, Jais P, Shah DC, et al. Spontaneous initiation of atrial fibrillation by ectopic beats originating in the pulmonary veins. New Engl J Med 1998;339:659-666. DOI:10.1056/NEJM199809033391003

24. Chang SL, Lin YJ, Tai CT, et al. Induced atrial tachycardia after circumferential pulmonary vein isolation of paroxysmal atrial fibrillation: electrophysiological characteristics and impact of catheter ablation on the follow-up results. J Cardiovasc Electrophysiol 2009;20:388-394. DOI:10.1111/j.15408167.2008.01358.x

25. Takahashi S, Sueda T. Development of the Maze procedure and the contribution of Japanese surgeons. Gen Thorac Cardiovasc Surg. 2017;65:144-152. DOI:10.1007/s11748-016-0728-y

26. Stulak JM, Suri RM, Burkhart HM, et al. Surgical ablation for atrial fibrillation for two decades: are the results of new techniques equivalent to the Cox maze III procedure? J Thorac Cardiovasc Surg 2014;147:1478-1486. DOI: 10.1016/j.jtcvs.2013.10.084

27. Kajiyma T, Kondo Y, Ueda M, et al. Catheter ablation of atrial tachyarrhythmias after a Maze procedure: A single center experience. J Cardiol Cases 2018;19:89-92. DOI:10.1016/j.jccase.2018.12.003

Table 1. Baseline characteristics

\begin{tabular}{ll}
\hline Characteristics & Value \\
\hline Age, mean & $69.3 \pm 7.2$
\end{tabular}




\begin{tabular}{ll}
\hline Characteristics & Value \\
\hline Male sex, $\mathrm{n}(\%)$ & $20(54.1 \%)$ \\
Body mass index, $\mathrm{kg} / \mathrm{m}^{2}$ & $22.7 \pm 3.0$ \\
Left ventricular ejection fraction, $\%$ & $53.7 \pm 11.7$ \\
Left atrium diameter, mm & $46.9 \pm 7.7$ \\
Left atrium volume, $\mathrm{mL}$ & $110.5 \pm 32.5$ \\
Left atrium volume index, $\mathrm{mL} / \mathrm{m}^{2}$ & $69.6 \pm 20.5$ \\
$\mathrm{CHADS}_{2}$ score, mean & $2.1 \pm 1.2$ \\
$\mathrm{CHA}_{2} \mathrm{DS}_{2}$-VASc score, mean & $2.4 \pm 1.3$ \\
$\mathrm{Hematocrit}_{\text {Oem }}$ & $39.3 \pm 3.9$ \\
Serum Creatinine, mg/dL & $0.82 \pm 0.2$ \\
Brain natriuretic peptide, $\mathrm{pg} / \mathrm{dL}$ & $86.7 \pm 61.9$ \\
Anti-arrhythmia drugs use before ablation, $\mathrm{n}(\%)$ & $26(70.3 \%)$ \\
\hline
\end{tabular}

Table 2. Details of cardiac surgery and ablation.

\begin{tabular}{|c|c|c|c|c|c|c|c|c|}
\hline No. & Sex & Age & Surgery & Maze & $\begin{array}{l}\text { LAA } \\
\text { inter- } \\
\text { vention }\end{array}$ & $\begin{array}{l}\text { Maze } \\
\text { energy }\end{array}$ & $\begin{array}{c}\text { Acute } \\
\text { Tachycardiauccess }\end{array}$ & $\begin{array}{l}\text { Post- } \\
\text { ablation } \\
\text { AAD } \\
\text { use }\end{array}$ \\
\hline 1 & M & 74 & $\begin{array}{l}\text { LAA } \\
\text { resection }\end{array}$ & Cryo & Yes & Cryo & Yes & Yes \\
\hline 2 & $\mathrm{~F}$ & 74 & $\begin{array}{l}\text { MVR, } \\
\text { TAP }\end{array}$ & Cryo & Yes & Cryo & $\begin{array}{ll}\text { M- } & \text { Yes } \\
\text { AFL } & \text { (gap), } \\
\text { T- } & \\
\text { AFL } & \text { (gap) }\end{array}$ & Yes \\
\hline 3 & M & 69 & $\begin{array}{l}\text { MVR, } \\
\text { TAP }\end{array}$ & Cryo & Yes & Cryo & $\begin{array}{l}\text { M- } \\
\text { AFL (gap), } \\
\text { AF }\end{array}$ & No \\
\hline 4 & M & 76 & $\begin{array}{l}\text { AVR, } \\
\text { TAP, } \\
\text { CABG }\end{array}$ & Cryo & No & Cryo & $\begin{array}{l}\text { M- } \quad \text { Yes } \\
\text { AFL (gap) }\end{array}$ & Yes \\
\hline 5 & $\mathrm{~F}$ & 67 & $\begin{array}{l}\text { VSD } \\
\text { closure, } \\
\text { TAP }\end{array}$ & Cryo & Yes & Cryo & $\begin{array}{l}\text { M- } \\
\text { AFL(gap) }\end{array}$ & Yes \\
\hline 6 & $\mathrm{~F}$ & 74 & $\begin{array}{l}\text { MVR, } \\
\text { AVR }\end{array}$ & Cryo & Yes & Cryo & $\begin{array}{l}\text { T- } \quad \text { Yes } \\
\text { AFL (gap) }\end{array}$ & Yes \\
\hline 7 & $\mathrm{~F}$ & 70 & MVP & Cryo & No & Cryo & $\begin{array}{l}\text { M- Yes } \\
\text { AFL (gap), } \\
\text { LAA } \\
\text { AT (gap), } \\
\text { RA M- } \\
\text { AT (gap) }\end{array}$ & No \\
\hline 8 & $\mathrm{~F}$ & 74 & $\begin{array}{l}\text { MVP, } \\
\text { TAP }\end{array}$ & $\begin{array}{l}\text { Maze } \\
\text { IV }\end{array}$ & Yes & $\mathrm{RF}$ & $\begin{array}{l}\text { M- Yes } \\
\text { AFL (gap), } \\
\text { LAA } \\
\text { AT (gap), }\end{array}$ & Yes \\
\hline
\end{tabular}




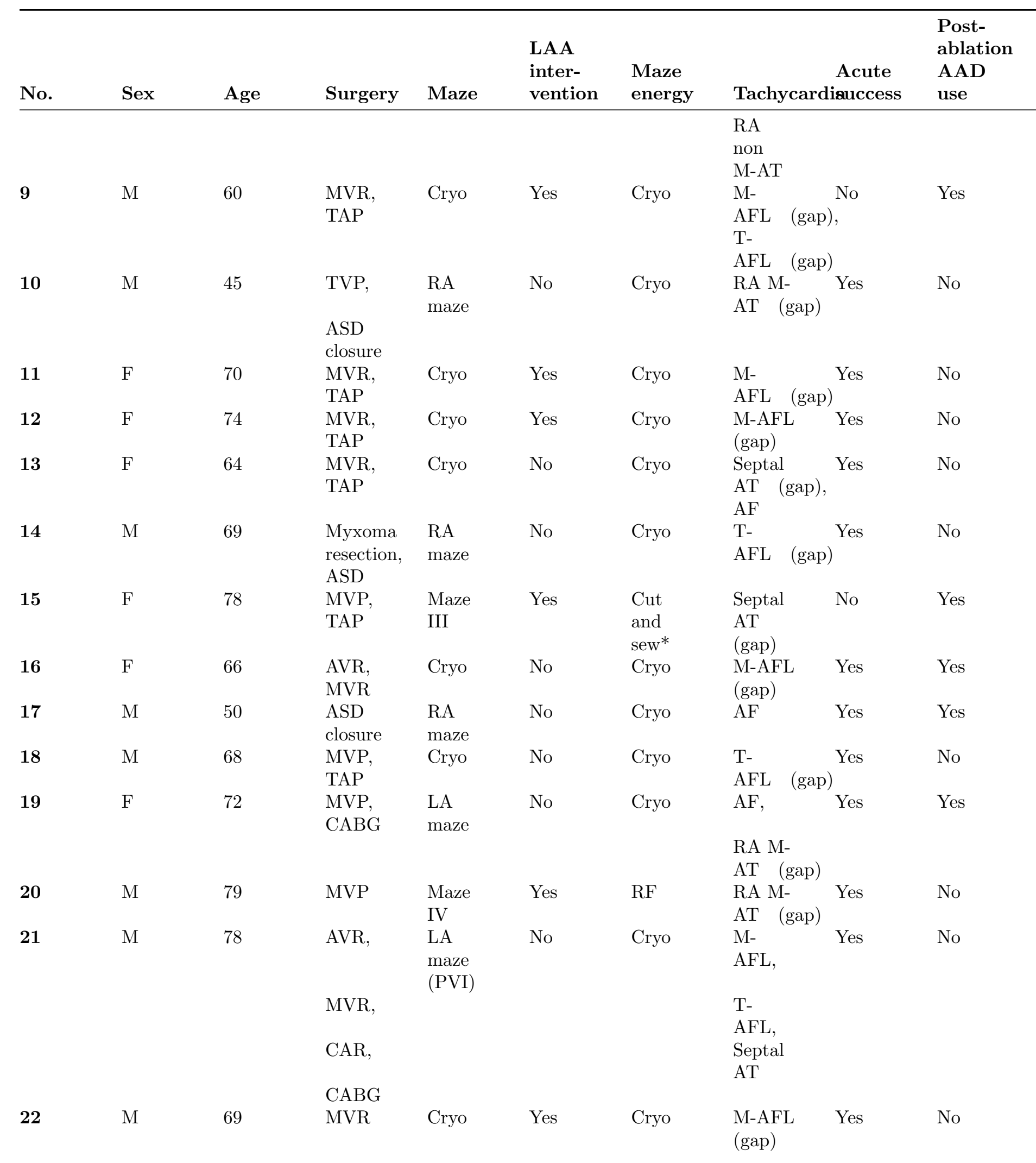




\begin{tabular}{|c|c|c|c|c|c|c|c|c|c|}
\hline No. & Sex & Age & Surgery & Maze & $\begin{array}{l}\text { LAA } \\
\text { inter- } \\
\text { vention }\end{array}$ & $\begin{array}{l}\text { Maze } \\
\text { energy }\end{array}$ & Tachyca & $\begin{array}{l}\text { Acute } \\
\text { isuccess }\end{array}$ & $\begin{array}{l}\text { Post- } \\
\text { ablation } \\
\text { AAD } \\
\text { use }\end{array}$ \\
\hline 23 & $\mathrm{~F}$ & 79 & $\begin{array}{l}\text { MVR, } \\
\text { TAP, } \\
\text { AVP }\end{array}$ & Cryo & Yes & $\mathrm{RF}$ & $\begin{array}{l}\text { M-AFL } \\
\text { (gap) }\end{array}$ & Yes & Yes \\
\hline 24 & M & 61 & $\begin{array}{l}\text { ASD } \\
\text { closure } \\
\text { TAP }\end{array}$ & Cryo & Yes & Cryo & $\begin{array}{l}\text { LAA } \\
\text { AT } \\
\text { (gap), } \\
\text { AF }\end{array}$ & Yes & Yes \\
\hline 25 & $\mathrm{M}$ & 67 & $\mathrm{CABG}$ & Cryo & No & Cryo & $\begin{array}{l}\text { M-AFL } \\
\text { (gap), } \\
\text { RA } \\
\text { non } \\
\text { M-AT, } \\
\text { T-AFL } \\
\text { (gap) }\end{array}$ & Yes & No \\
\hline 26 & $\mathrm{~F}$ & 69 & $\begin{array}{l}\text { MVR, } \\
\text { TAP }\end{array}$ & Cryo & Yes & Cryo & $\begin{array}{l}\text { M-AFL } \\
\text { (gap), } \\
\text { AF }\end{array}$ & Yes & Yes \\
\hline 27 & $\mathrm{M}$ & 68 & $\begin{array}{l}\text { MVR, } \\
\text { TAP }\end{array}$ & $\begin{array}{l}\text { Maze } \\
\text { III }\end{array}$ & Unknown & $\begin{array}{l}\text { Cut } \\
\text { and } \\
\text { sew* }\end{array}$ & $\begin{array}{l}\text { M-AFL } \\
\text { (gap), } \\
\text { LA non } \\
\text { M-AT, } \\
\text { Septal } \\
\text { AT } \\
\text { AF }\end{array}$ & Yes & Yes \\
\hline 28 & M & 70 & $\begin{array}{l}\text { VSD } \\
\text { closure }\end{array}$ & $\begin{array}{l}\text { Maze } \\
\text { III }\end{array}$ & Yes & $\begin{array}{l}\text { Cut } \\
\text { and } \\
\text { sew* }\end{array}$ & $\begin{array}{l}\text { M-AFL } \\
\text { (gap), } \\
\text { T-AFL } \\
\text { (gap) }\end{array}$ & Yes & No \\
\hline 29 & $\mathrm{~F}$ & 73 & $\begin{array}{l}\text { MVR, } \\
\text { TAP }\end{array}$ & $\begin{array}{l}\text { Maze } \\
\text { III }\end{array}$ & Yes & $\mathrm{RF}$ & $\begin{array}{l}\text { LAA } \\
\text { AT } \\
\text { (gap), } \\
\text { T-AFL } \\
\text { (gap) }\end{array}$ & Yes & Yes \\
\hline 30 & M & 63 & AVR & $\begin{array}{l}\mathrm{LA} \\
\text { maze }\end{array}$ & No & Cryo & $\begin{array}{l}\text { M-AFL } \\
\text { (gap) }\end{array}$ & Yes & No \\
\hline 31 & M & 73 & $\begin{array}{l}\text { MVP, } \\
\text { CABG }\end{array}$ & Kosakai & Unknown & $\begin{array}{l}\text { Cut } \\
\text { and } \\
\text { sew* }\end{array}$ & $\mathrm{AF}$ & Yes & No \\
\hline 32 & $\mathrm{~F}$ & 71 & MVP & $\begin{array}{l}\text { Maze } \\
\text { IV }\end{array}$ & No & $\mathrm{RF}$ & $\begin{array}{l}\text { M- } \\
\text { AFL, } \\
\text { AF }\end{array}$ & Yes & Yes \\
\hline 33 & $\mathrm{~F}$ & 63 & MVR & Cryo & Yes & Cryo & $\begin{array}{l}\text { M-AFL } \\
\text { (gap) }\end{array}$ & Yes & No \\
\hline 34 & M & 56 & $\begin{array}{l}\text { AR, } \\
\text { ADR }\end{array}$ & Cryo & Yes & Cryo & $\mathrm{AF}$ & Yes & Yes \\
\hline
\end{tabular}




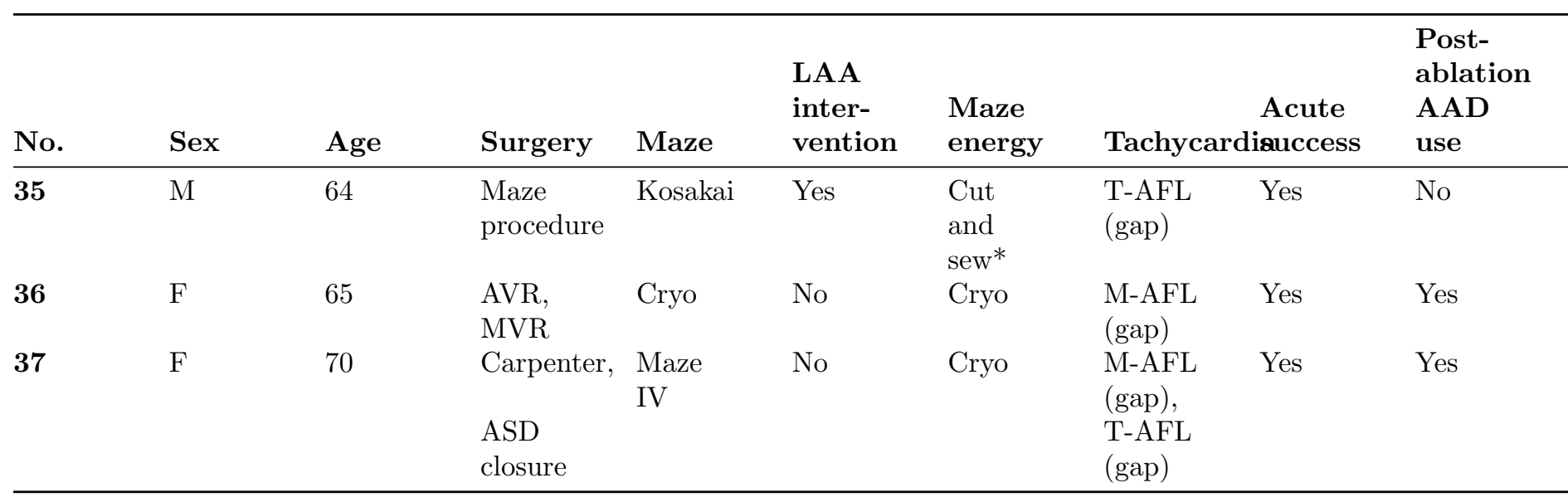

$\mathrm{AAD}$, anti-arrhythmia drug; ADR, aortic dissection repair; AF, atrial fibrillation; AR, aortic replacement; ASD, atrial septal defect; AT, atrial tachycardia; AVR, aortic valve replacement; CABG, coronary artery bypass graft; CAR, coronary aneurysm resection; Chem, chemical ablation; LA, left atrium; LAA, left atrium appendage; M-AT, macroreentrant-atrial tachycardia; M-AFL, peri-mitral atrial flutter; MVP, mitral valve plasty; MVR, mitral valve replacement; PVI, pulmonary vein isolation; RA, right atrium; RF, radiofrequency; TAP, tricuspid valve plasty; T-AFL, cavotricupid isthmus dependent atrial flutter; TVP, tricuspid valve replacement; VSD, ventricular septal defect.

(gap), gap-related; *, partial using cryo energy.

Table 3. Number of arrhythmias for each Maze procedure

\begin{tabular}{lllll}
\hline & Cryo Maze (21 cases) & Maze IV (4 cases) & Kosakai's Maze (2 case) & Maze III (4 ca \\
\hline Left M-AT (n) & 15 & 3 & 0 & 2 \\
Right M-AT (n) & 6 & 2 & 1 & 2 \\
Left non-M-AT (n) & 2 & 2 & 0 & 1 \\
Right non-M-AT (n) & 1 & 1 & 0 & 0 \\
Septal AT (n) & 1 & 0 & 0 & 2 \\
AF (n) & 6 & 1 & 1 & 1 \\
\hline
\end{tabular}

AF, atrial fibrillation; AT, atrial tachycardia; F, focal; LA, left atrial; M, macroreentrant; RA, right atrial.

Table 4. Relationship among PV gap, the occurrence of PV gap repeated AT and AF, and the Maze procedure.

\begin{tabular}{lllll}
\hline & Maze III (4 cases) & Kosakai' s Maze (2 cases) & Kosakai' s Maze (2 cases) & Cryo Maze \\
\hline PV gap (n) & 2 & 1 & 9 & 9 \\
AF or PV gap repeated AT (n) & $2^{*}$ & $1^{* *}$ & $6^{* *}$ & $6^{* *}$ \\
\hline
\end{tabular}

*, PV gap related AT; **, AF

\begin{tabular}{llll}
\hline & Cut and sew (5 cases) & Non-cut and sew (29 cases) & P \\
\hline PV gap (case) & 2 & 17 & 0.44 \\
AF or PV gap repeated AT (case) & 2 & 8 & 0.15 \\
\hline
\end{tabular}


Cut and sew include Maze III and Kosakai's Maze, Non-cut and sew include Cryo Maze, Maze IV and LA Maze, PV: pulmonary vein

Defined as significant at $\mathrm{P}<0.05$

\begin{tabular}{lll}
\hline & AF or PV gap related AT were induced case (10 cases) & AF or PV gap related AT were not induced ca \\
\hline PV gap positive & 10 & 10 \\
PV gap negative & 0 & 12 \\
\hline
\end{tabular}

$\mathrm{P}=0.003$ with significant difference

AF, atrial fibrillation; AT, atrial tachycardia; LA, left atrium; PV, pulmonary vein, RA, right atrium.

*The 2 of 24 cases were not PV gap checked because only RA side AT was induced.

Figure legends

Fig 1. The classification of atrial arrhythmia.

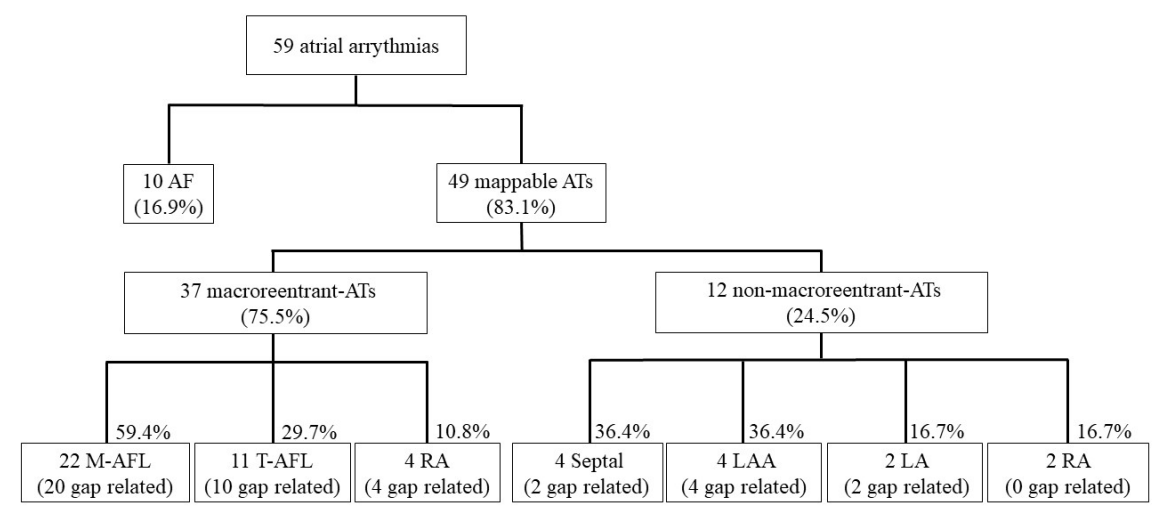

This figure shows the classification of atrial arrhythmia in this study. Fifty-nine atrial arrhythmias were divided into 49 mappable ATs and 10 AF. Mappable ATs are divided into 37 macroreentant-AT and 12 nonmacroreentant-AT. Macroreentant-ATs are divided into $22 \mathrm{M}$-AFL, 11 T-AFL, and 4 RA macroreentant-ATs. Non-macroreentant-ATs are divided into 4 septal, 4 LAA, 2 LA, and 2 RA focal ATs. Each ATs are stated whether these are gap-related or not.

AF, atrial fibrillation; AT, atrial tachycardia; LA, left atrium; LAA, left atrium appendage; M-AFL, perimitral atrial flutter; RA, right atrium; T-AFL, cavotricupid isthmus dependent atrial flutter.

Fig 2. The number of residual electrical conductions (gap) sites after all Maze procedure (37 cases). 


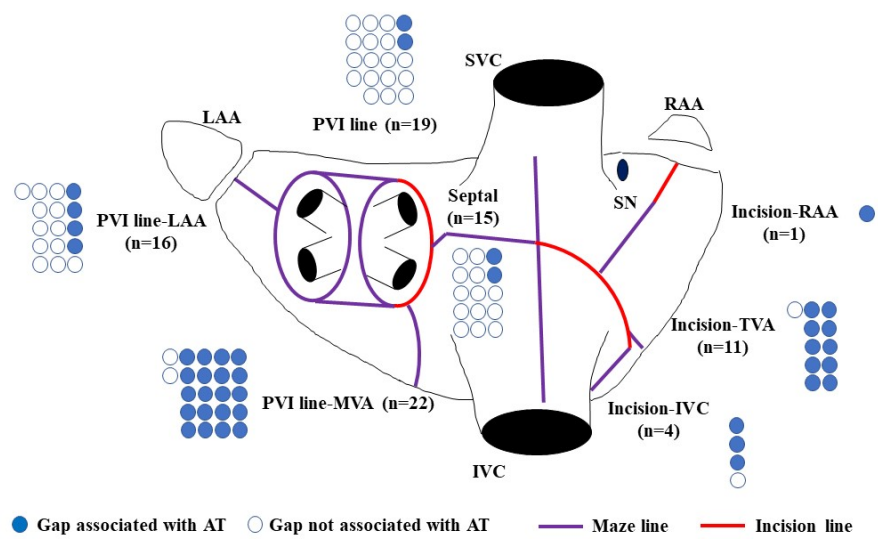

This figure shows all the Maze lines in this study. The Maze procedures include Maze III, Kosakai's Maze, Maze IV, Cryo Maze, LA Maze, and RA Maze. The Blue blue circle indicates the gap associated with AT, the white circles indicate the gap not associated with AT, the purple line indicates the Maze line which made by RF or Cryo or energy, and the red line indicates incision line.

IVC, inferior vena cava; LAA, left atrial appendage; MVA, mitral valve annulus; PVI, pulmonary vein isolation; RAA, right atrial appendage; SN, sinus node; SVC, superior vena cava; TVA, tricuspid valve annulus.

Fig3. A representative case (Case 7) of 3 forms of gap related ATs after CryoMaze procedure.

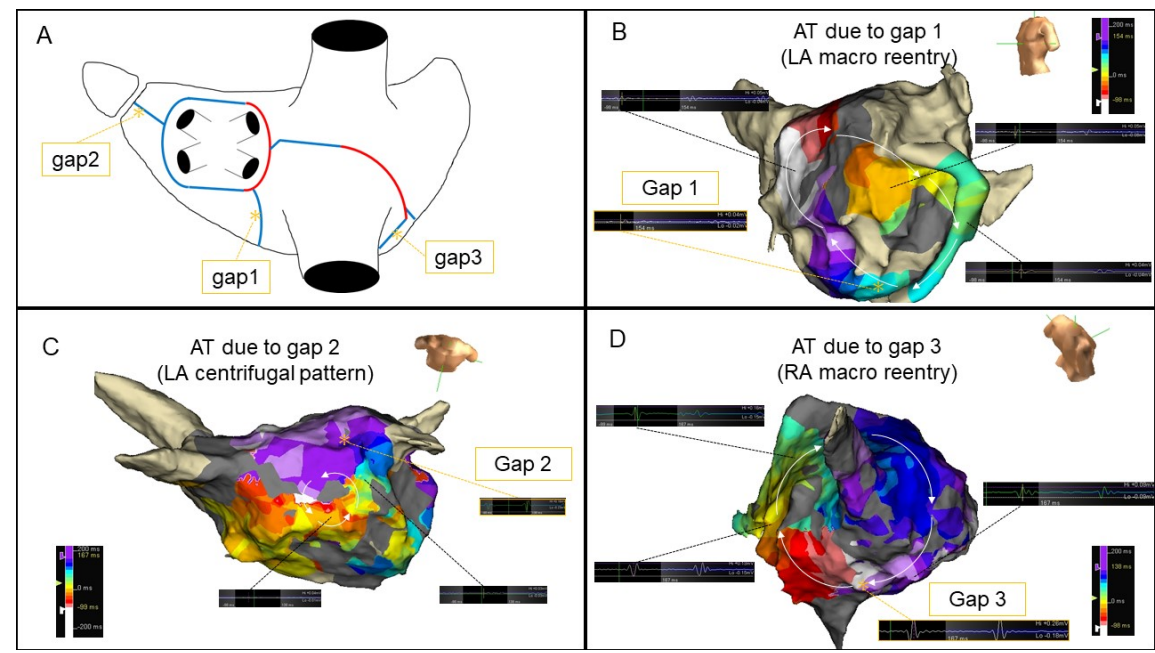

A: Lines of the CryoMaze procedure performed in our institution. ${ }^{2}$ Blue lines indicate using cryo energy, red lines indicate incision, and orange astarisks indicate gap in this case. B: This figure shows activation map of clockwise rotation M-AFL which related to the gap between PVI line and mitral valve anulus (gap 1). C: This figure shows activation map of LAA AT which related to the gap between PVI line and LAA (gap 2). D: This figure shows activation map of RA M-AT which related to the gap between RA incision and IVC (gap 3). 
AT, atrial tachycardia; IVC, inferior vena cava; M-AFL, peri-mitral atrial flutter; PVI, pulmonary vein isolation; LAA, left atrial; LAA, left atrial appendage; RA, right atrial.

Fig 4.

The progress of 37 consecutive cases in this study.

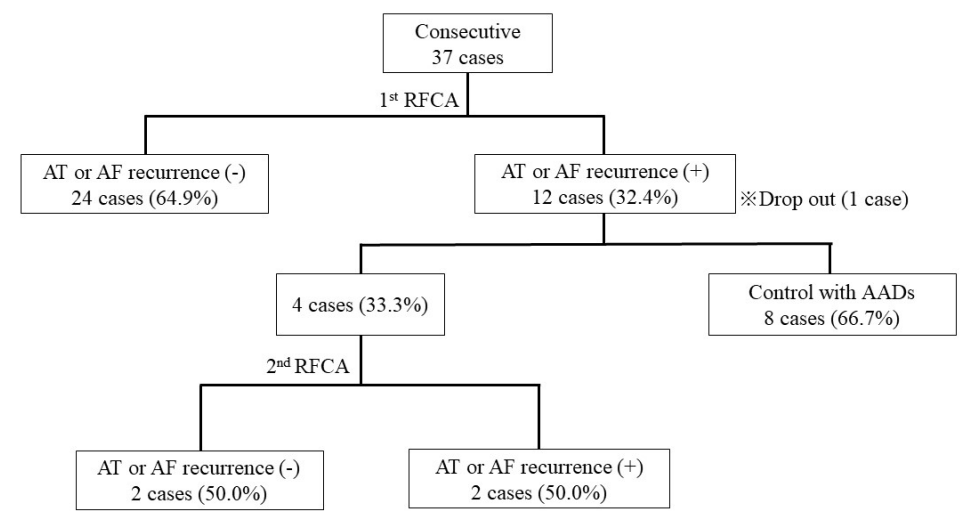

Twelve of 37 consecutive cases had a recurrence after the first catheter ablation, 24 cases did not have a recurrence and 1 case was unable to be followed up because of the loss of contact. Four of the 12 cases underwent catheter ablation twice. Two cases had the same AT as the first session, while the other 2 had were no recurrence after the second session.

AAD, antiarrhythmic drug; AF, atrial fibrillation; AT, atrial tachycardia; RFCA, radio frequency catheter ablation. 